\title{
Assessing the integrity of CFRPs through nanomechanical mapping: the effect of CF surface modification
}

\author{
Elias P. Koumoulos ${ }^{1}$, Panagiotis Kainourios ${ }^{1}$, and Costas A. Charitidis ${ }^{1, *}$ \\ ${ }^{1}$ Research Unit of Advanced, Composite, Nano-Materials and Nanotechnology (R-Nano Lab), \\ Material Science and Engineering Department, School of Chemical Engineering, National Technical \\ University of Athens, Zographou, GR-15780, Greece
}

\begin{abstract}
The purpose of this study is to assess the effect of CF surface modification in enhancement of the wetting properties of carbon fibers in order to improve the adhesion force between the fiber and the polymer matrix; for this, the integrity of CFRPs through nanomechanical mapping was evaluated. The surface of commercial carbon fibers was functionalized through cyclic voltammetry in aqueous electrolyte solutions of $\mathrm{H}_{2} \mathrm{SO}_{4}$, in the presence of acrylic acid, methacrylic acid, acrylonitrile and $\mathrm{N}$ vinylpyrrolidone monomers. The produced surface modified carbon fibers were embedded in epoxy resin. Elastic modulus nanoindentation mapping was performed in order for elastic modulus to be calculated, as a qualitative assessment of fibre - matrix interaction. For this, a grid protocol was set up for the integrity assessment of CFRPs through nanomechanical mapping.
\end{abstract}

\section{Introduction}

Over the last decade carbon fiber (CF) has been considered as one of the most promising reinforcement materials, while epoxy based resins or thermoplastic polymers are mainly utilised as matrices for the synthesis of composite materials, meeting a large variety of application requirements, i.e. from automobile to aerospace, medical and structural operations. Carbon fiber composites exhibit enhanced mechanical performances, such as extremely high strength and modulus, good stiffness, and creep resistance etc. [1-2]. However, according to the literature [3], due to lack of fiber-matrix strong chemical bonding, the application of stress to the carbon fiber composite results in poor mechanical properties, e.g. decreased interlaminar shear strength (ILSS) and toughness, as stress transfer from one carbon filament to another occur via the matrix material [3]; thus, $\mathrm{CF}$ is pulled out of the matrix (pull-out effect).

This drawback is confronted with several approaches of surface treatment in order to introduce active groups on the fibers surface that will enhance the bonding with the matrix resin. It is noticed that if the bond is too strong, it will result in a brittle composite, whereas if the treatment is feeble the malfunction will remain the same. Evidently, it is of high

\footnotetext{
* Corresponding author: charitidis@chemeng.ntua.gr
} 
importance to establish an optimum level of surface treatment for a given fiber and matrix system [3-4].

A wide variety of surface treatments have been introduced in order to overcome the issue at hand, e.g. gas phase oxidation treatment, liquid phase oxidation treatment, plasma treatment as well as electrochemical treatment [5]. The effects of surface treating methods of T700 grade high-strength carbon fiber GQ4522 and the interfacial bonding between the fiber with epoxy resin matrix are reported in literature [6], including evaluation of sizing and heat treatment on physical and chemical properties as well as electrolysis of anodic oxidation. Surface chemical treatment is also considered of importance for the formation of composite interface (CF-matrix) and adjusting interfacial properties; using plasma oxidation, Lee et al. [7] investigated the effects of plasma surface treatment of recycled CF on interfacial after various treatment times. Modified commercial CF using chemical and heat treatment in order to obtain CF with improved catalytic properties, are also investigated [8], while electrochemical and ultra-violet/ozone process are reported for functionalisation of polyacrylonitrile (PAN) based CF surface [9].

Nanoindentation testing has emerged as a convenient and fast method for the assessment of localized surface mechanical properties. In this technique, a diamond tip of known geometry is directed into a sample surface while the applied load and the resulting depth values are recorded simultaneously and loop-controlled through actuators and sensors [10]. The hardness (measured as the resistance to contact pressure) and the elastic modulus of the material being tested is then extracted from the first segment of the unloading curve, assuming an elastic behaviour using the theory of contact mechanics [11]. The standard method is capable of providing information on the elastic recovery and plastic deformation [12], with unprecedented lateral and sub-surface displacement resolution. Additionally, nanoindentation may also be used in measuring mechanical properties such as fracture behaviour [13], hardening, strain rate sensitivity, residual stress [14] and size effects in plasticity on a local level [15].

\section{Experimental}

\subsection{Materials}

Carbon fibers were obtained from Toray Industries Inc. (Tokyo, Japan). Epoxy resin Araldite GY 257 (Jubail Chemical Industries, JANA, Kingdom of Saudi Arabia), 2,2'Diaminodiethylamine (HY 943, Sigma-Aldrich) and N,N-Methylenebis(acrylamide) (Acros Organics) were used without further purification. Acrylic acid (Acros Organics), methacrylic acid (Acros Organics), acrylonitrile (Acros Organics) and N-vinylpyrrolidone (Acros Organics) were double distilled under reduced pressure prior to use. The specifications of the obtained CFs and epoxy resin are reported in literature [1].

\subsection{Characterisation Methods}

The indentation results provide significant information under localised compression; for carbon nanotube forest use e.g. as super-compressive cushions, electrical contacts and switches, or thermal conduits, knowing the applied displacements is necessary to identify the extent of the buckling region which acts to provide energy absorption [11]. The nanoindentation tests were performed using a Hysitron TriboLab ${ }^{\circledR}$ Nanomechanical Test Instrument equipped with a Berkovich diamond indenter (average radius $100 \mathrm{~nm}$ ) which allows the application of loads from 1 to $30000 \mu \mathrm{N}$ and records the displacement as a 
function of applied loads with a high load resolution $(1 \mathrm{nN})$ and a high displacement resolution $(0.04 \mathrm{~nm})$. The nanomechanical test instrument employed in this study is equipped with a SPM (for imaging, a sharp Berkovich diamond indenter of 50nm radius was used), in which a sharp probe tip moves in a raster scan pattern across a sample surface using a three-axis piezo positioner. Details about the instrument and the experimental setup have been presented elsewhere [16]. Prior to indentation, the area function of the indenter tip was calibrated in a fused silica and in a soft PS-6 sheet $\left(E_{r}=0.007 \mathrm{GPa}(7 \mathrm{MPa})\right)$ for shallow depths, standard materials for this purpose; getting modulus and hardness values from small $(\sim 4-5 \mathrm{~nm})$ indentation depths requires a very careful calibration of the tip. All nanoindentation tests were conducted in a clean area environment with $45 \%$ humidity and $23^{\circ} \mathrm{C}$ ambient temperature, at $200 \mathrm{~nm}$ of displacement, with displacement feedback control closed loop [16].

\subsection{Surface Treatment and Composite Manufacturing}

All electropolymerization experiments were performed at room temperature, in onecompartment electrochemical cell of $150 \mathrm{~mL}$ volume consisting of the carbon fiber as a working electrode $\left(\approx 1.0 \mathrm{~cm}^{2}\right.$ of exposed area), a saturated silver chloride electrode $[\mathrm{Ag} / \mathrm{AgCl}, \mathrm{KCl}(\mathrm{sat})]$ as reference and a platinum foil as counter electrode. The electropolymerization solution was deoxygenated by bubbling nitrogen for 10 minutes prior to the electropolymerization process. The produced coated carbon fiber bundle was thoroughly rinsed with water and acetone to remove residues and was placed to dry into an oven [1]. The electrografting of each polymer onto the fiber surface resulted in the production of unidirectional (UD) composite. Subsequently, the polymer coated carbon fiber bundles were placed inside dumb bell type sacrificial templates and then the epoxy resin solution was added to each template. The dimensions of the templates were selected in agreement with ASTM D638-14. The synthetic process for the epoxy resin solution was followed according to our previous work [17]. Briefly, both resin GY 257 and 2,2'Diaminodiethylamine were added slowly in acetone solvent, under vigorous stirring and the obtained mixture was left to cure at room temperature. The wt.\% material concentration of the epoxy solution is reported in literature previous work [1].

\section{Results and Discussion}

Typical load-depth response of the CFRPs were performed following a trapezoidal loading and unloading curve; loading and unloading segment time was $40 \mathrm{~s}$ each, and holding time was set at $3 \mathrm{~s}$.
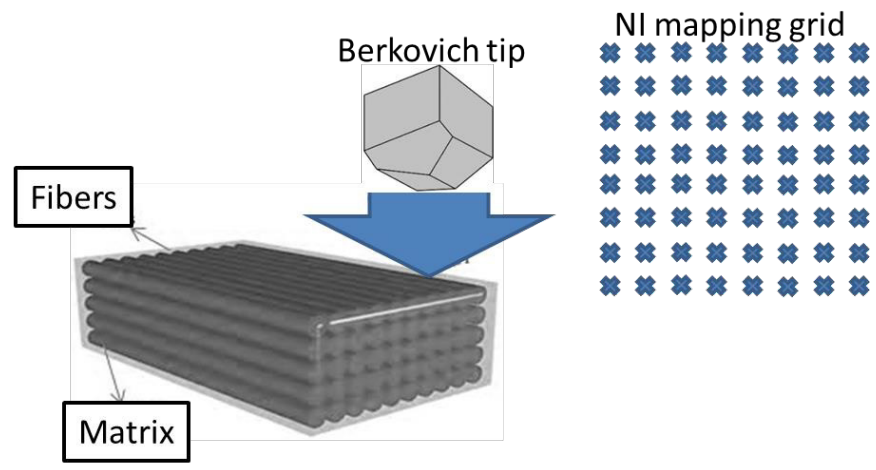

Figure 1. Nanoindentation (NI) schematic representation of mapping grid measurements on top of unidirectional (UD) composite samples. 


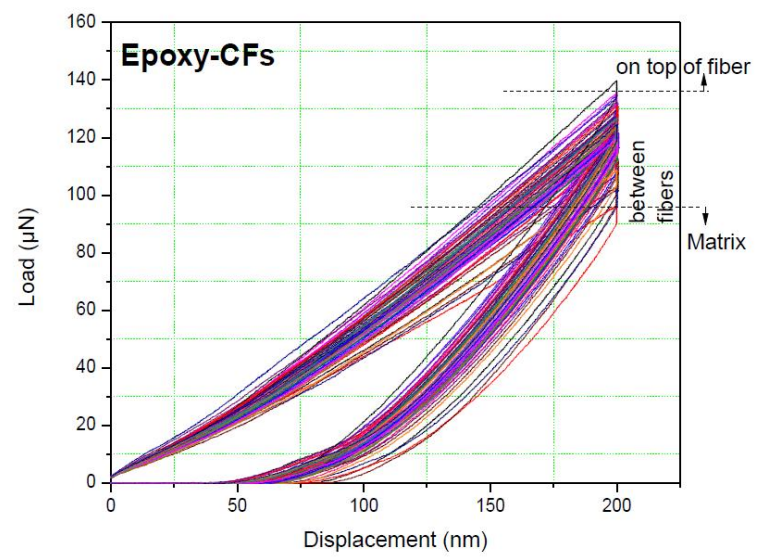

Figure 2. Load-displacement data from the loading-unloading mapping measurements at $200 \mathrm{~nm}$ of displacement, for epoxy-CF plain samples.

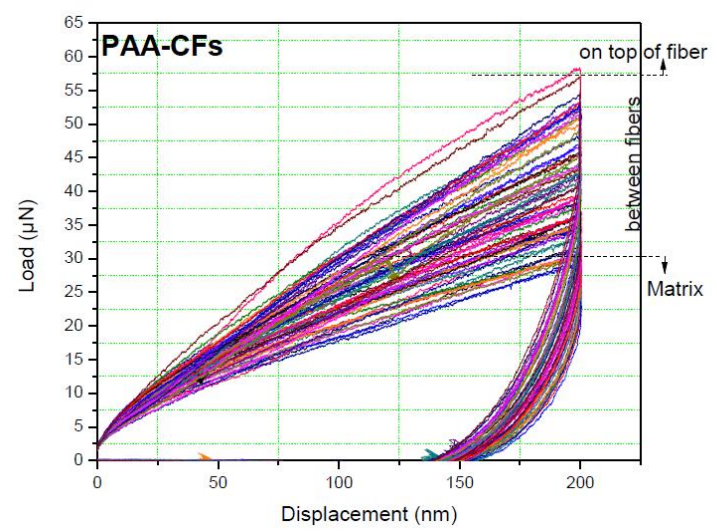

Figure 3. Load-displacement data from the loading-unloading mapping measurements at $200 \mathrm{~nm}$ of displacement, for epoxy-CF/PAA modified samples.

In Figs. 2-5, load-displacement data from the loading-unloading cycles at $200 \mathrm{~nm}$ of displacement, for all samples, are presented. A measure of the plasticity of a material is given by the "plasticity index" which, in the case of nanoindentation, can be taken as the ratio of the area enclosed between the loading-unloading curves to the area under the loading part of the curve. The obtained results reveal that for the case of PAA-CFs, an increased plastic deformation is recorded (together with viscoelasticity and adhesiondiscontinuities and fluctuations during measurement), while for the case of epoxy-CFs a load range of $40 \mu \mathrm{N}$ is revealed, together with decreased plastic deformation (PAN-CFs and PMAA CFs with a load range of $\sim 55 \mu \mathrm{N}$ and $\sim 90 \mu \mathrm{N}$, respectively). Nanoindentation tends to produce relatively high local stresses and it is fairly common experience for these to generate obvious time-dependent effects, such as progressive indenter penetration when a constant load is maintained. 


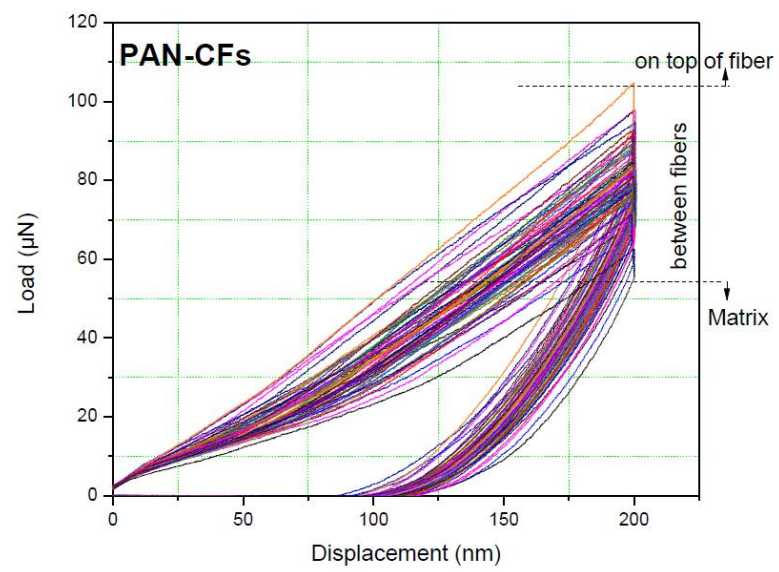

Figure 4. Load-displacement data from the loading-unloading mapping measurements at $200 \mathrm{~nm}$ of displacement, for epoxy-CF/PAN modified samples.

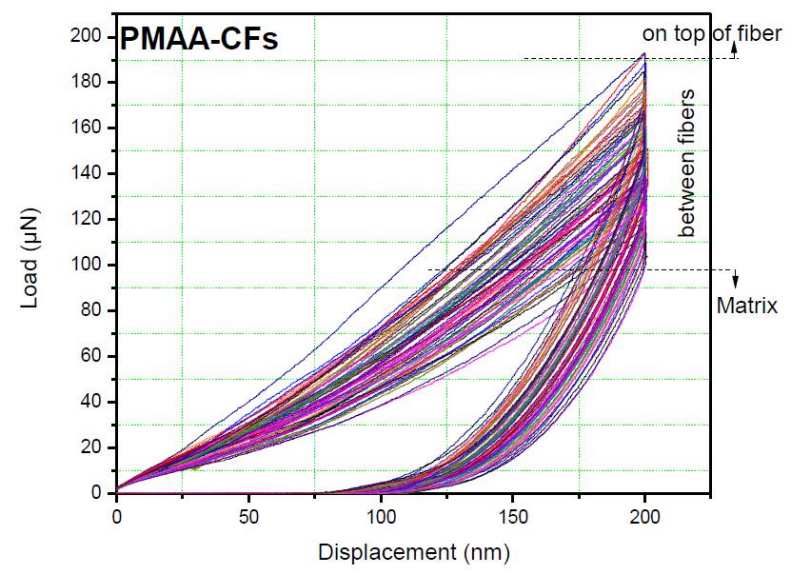

Figure 5. Load-displacement data from the loading-unloading mapping measurements at $200 \mathrm{~nm}$ of displacement, for epoxy-CF/PMAA modified samples.

Consequently, it will be quite often necessary to take into account the possibility of creep affecting the results when experimental nanoindentation data are to be used to obtain constitutive relations. Figure 3 shows the loading-unloading curves for PAA-CFs samples, which exhibit interesting local discontinuities measured in the load-controlled test of this study; these are characteristic of energy-absorbing or energy-releasing events occurring beneath the indenter tip. However, the transition from purely elastic to elastic/plastic deformation, that is gradual slope change (yield-type "pop-in"), is observed for the case of plain PAN-CFs at $\sim 20 \mathrm{~nm}$. As nanoindentation is a localized process, an increase in local crystallinity can also lead to modulus values increase.

Elastic modulus mapping for all samples at $200 \mathrm{~nm}$ of displacement revealed that for the case of PMAA-CFs, increased values $(\sim 3.5 \mathrm{GPa})$ are recorded; this is also consistent with the data of figure 5, as it is evident that higher applied loads are required for reaching the displacement of $200 \mathrm{~nm}$, when compared with all other samples (for which the maximum elastic modulus values were found $\sim 1.7 \mathrm{GPa}$ ). 


\section{Conclusions}

The purpose of this study was to assess the effect of CF surface modification in enhancement of the wetting properties of carbon fibers in order to improve the adhesion force between the fiber and the polymer matrix; for this, the integrity of CFRPs through nanomechanical mapping was evaluated. The surface of commercial carbon fibers was functionalized; then, the produced surface modified carbon fibers were embedded in epoxy resin. Elastic modulus nanoindentation mapping for all samples at $200 \mathrm{~nm}$ of displacement revealed that for the case of PMAA-CFs, increased values $(\sim 3.5 \mathrm{GPa})$ are recorded; it is also evident that higher applied loads are required for reaching the displacement of $200 \mathrm{~nm}$ for the case of PMAA-CFs, when compared with all other samples (for which the maximum elastic modulus values were found $\sim 1.7 \mathrm{GPa}$ ).

Overall, grid protocol was proved as a powerful tool for the integrity assessment of CFRPs through nanomechanical mapping, revealing the effect extent of CF surface modification.

This work was supported by the EU H2020 Project "Modified Cost Effective Fibre Based Structures with Improved Multi-Functionality and Performance" (MODCOMP) under Grant Agreement no. 685844.

\section{REFERENCES}

1. P. Kainourgios, I. Kartsonakis, D. Dragatogiannis, E. P. Koumoulos, P. Goulis, C. A. Charitidis, App. Sur. Sci. 416, (2017)

2. X. Yuan, B. Zhu, X. Cai, J. Liu, K. Qiao, J. Yu, App. Sur. Sci. 401 (2017)

3. P. Morgan, CRC Press, Taylor \& Francis Group, Boca Raton, 2005, pp. 347-376.

4. Y. Zhang, Y. Zhang, Y. Liu, X. Wang, B. Yang, App. Sur. Sci. 382 (2016)

5. S. Tiwari, J. Bijwe, S. Panier, Wear 274-275, (2012).

6. Q. Ma, Y. Gu, M. Li, S. Wang, Z. Zhang, App. Sur. Sci. 379 (2016)

7. H. Lee, I. Ohsawa, J. Takahashi, App. Sur. Sci. 328 (2015)

8. S. Yang, L. Li, T. Xiao, D. Zheng, Y. Zhang, App. Sur. Sci. 383 (2016)

9. S. Osbeck, S. Ward, H. Idriss, App. Sur. Sci. 270 (2013)

10. E. P. Koumoulos, S. A. M. Tofail, C. Silien, D. De Felicis, R. Moscatelli, D. A. Dragatogiannis, E. Bemporad, M. Sebastiani, C. A. Charitidis, Mat. Des. 137 (2018)

11. E.P. Koumoulos, C. Charitidis, Appl. Surf. Sci. 396 (2017)

12. E.P. Koumoulos, C.A. Charitidis, D.P. Papageorgiou, A.G. Papathanasiou, A.G. Boudouvis, Surf. Coat. Technol. 206, 19 (2012)

13. E.P. Koumoulos, P. Jagdale, I.A. Kartsonakis, M. Giorcelli, A. Tagliaferro, C.A. Charitidis, Polym. Compos. 36, 8 (2015)

14. C.A. Charitidis, D.A. Dragatogiannis, E.P. Koumoulos, I.A. Kartsonakis, Mater. Sci. Eng. A 540 (2012)

15. C.A. Charitidis, E.P. Koumoulos, D.A. Dragatogiannis, Lubr. 1 (2) (2013)

16. E.P. Koumoulos, P. Jagadale, A. Lorenzi, A. Tagliaferro, C.A. Charitidis, Comp. Part B: Eng. 80 (2015)

17. I.A. Kartsonakis, A.C. Balaskas, E.P. Koumoulos, C.A. Charitidis, G.C. Kordas, Cor. Sci. 57 (2012) 